\title{
Utility of noncontrast MRI in the detection and risk grading of gastrointestinal stromal tumor: a comparison with contrast-enhanced CT
}

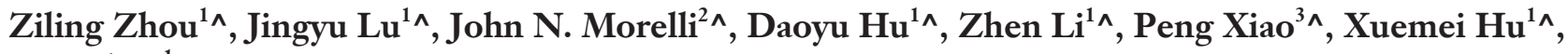 \\ Yaqi Shen ${ }^{1 \wedge}$
}

${ }^{1}$ Department of Radiology, Tongji Hospital, Tongji Medical College, Huazhong University of Science and Technology, Wuhan, China; ${ }^{2}$ Department of Radiology, St. John's Medical Center, Tulsa, OK, USA; ${ }^{3}$ Biomedical Engineering Department, Huazhong University of Science and Technology, Wuhan, China

Correspondence to: Yaqi Shen, MD, PhD. Department of Radiology, Tongji Hospital, Tongji Medical College, Huazhong University of Science and Technology, 1095 Jiefang Avenue, Wuhan 430030, China. Email: yqshen@hust.edu.cn.

Background: Recently developed adjuvant therapies for gastrointestinal stromal tumor (GIST) have been shown to improve patient survival. Guidelines currently recommend contrast-enhanced computed tomography (CECT) for GIST detection and surveillance. Patients with moderate-to-high risk GISTs require more frequent surveillance due to a higher 5-year recurrence rate. Our study aimed to compare noncontrast magnetic resonance imaging (MRI) with CECT for GIST detection, and evaluate volumetric apparent diffusion coefficients (ADCs) for risk stratification of GIST.

Methods: We retrospectively enrolled 83 patients with histopathologically confirmed GISTs for lesion detection efficiency analysis between noncontrast MRI and matched CECT studies. A 5-point scale was used by two independent reviewers to determine if the lesion was present or absent. Another cohort, comprising 28 patients with pathologically confirmed primary GISTs, was further screened for risk stratification, with a comparison of volumetric ADC parameters between the pathologically very-low-to-low risk and moderateto-high risk GIST patients.

Results: For identifying GISTs, the sensitivity and specificity of noncontrast MRI were $83.6 \%$ and $89.3 \%$ for reader 1 respectively, and $81.8 \%$ and $92.9 \%$ for reader 2 respectively; the sensitivity and specificity of CECT were $76.4 \%$ and $89.3 \%$ for reader 1 respectively, and 76.4 and $78.6 \%$ for reader 2 respectively. Tumor volumetric $\mathrm{ADC}$ histogram parameters, including $\mathrm{ADC}_{\max }, \mathrm{ADC}_{\text {stder }}, 90^{\text {th }}$ and $95^{\text {th }}$ percentiles, inhomogeneity, and entropy, were positively correlated with a higher risk grade of GIST (r=0.421-0.758). The receiver operator characteristic curve analysis showed $\mathrm{ADC}_{\max }$ achieved the highest area under the curve value of 0.938 for discriminating very-low-to-low risk versus moderate-to-high risk GISTs.

Conclusions: Noncontrast MRI was an efficient technique for identifying GIST patients. The combination of CECT and noncontrast MRI can improve the reliability of diagnosis. For patients with contraindications to CECT, noncontrast MRI may be a comparable alternative. Volumetric ADC histogram parameters may be useful in differentiating very-low-to-low risk from moderate-to-high risk primary GISTs.

Keywords: Diffusion magnetic resonance imaging (diffusion MRI); gastrointestinal stromal tumor (GIST); magnetic resonance imaging (MRI); multidetector computed tomography (multidetector CT)

\footnotetext{
^ ORCID: Ziling Zhou, 0000-0002-9793-8382; Jingyu Lu, 0000-0001-7364-9693; John N. Morelli, 0000-0002-6451-6984; Daoyu Hu, 0000-0001-9281-8552; Zhen Li, 0000-0002-9289-6785; Peng Xiao, 0000-0003-4991-0921; Xuemei Hu, 0000-0001-9009-0983; Yaqi Shen, 0000-0003-0589-8975.
} 
Submitted Apr 20, 2020. Accepted for publication Jan 27, 2021.

doi: 10.21037/qims-20-578

View this article at: http://dx.doi.org/10.21037/qims-20-578

\section{Introduction}

Gastrointestinal stromal tumor (GIST) is the most common mesenchymal neoplasm of the gastrointestinal tract, with an estimated annual incidence of $6.8-14.5$ cases per million (1). Adjuvant therapy has revolutionized the survival outcomes of metastatic GIST patients, with median survival duration having increased from approximately 20 months to more than 60 months $(2,3)$. Contrast-enhanced computed tomography (CECT) is the standard imaging modality for diagnosing GIST and assessing the therapeutic response of GIST (4). According to the latest version of the National Comprehensive Cancer Network guidelines, for patients with resectable GISTs, regular follow-up CECT can be performed up to 20 times in the first 5 years, and even more frequent follow-ups may be required for those with unresectable GISTs, recurrence, or metastatic GISTs (5). Only in patients with low-risk GISTs can the number of follow-up CECT be reduced. The dangers and concerns related to ionizing radiation in CT examinations, along with patients' desire to decrease ionizing radiation exposure, reduce patient compliance. The absence of ionizing radiation exposure and the foregoing of intravenous contrast agent administration make noncontrast magnetic resonance imaging (MRI) an increasingly flexible test in clinical practice that may potentially further improve the survival of GIST patients.

MRI has been introduced as an alternative or preferred imaging modality for gastrointestinal disease $(6,7)$. Recurrent tumors with a cyst-like appearance without enhancement may occur in a significant proportion of GIST patients during therapy (8). T2-weighted imaging (T2WI) can clearly delineate the boundary of a cystic GIST and its response to adjuvant therapies (9); meanwhile, diffusionweighted imaging (DWI) reflects pathological differences, including the risk grade of the primary GIST, through the calculation of apparent diffusion coefficient (ADC) values (10-13). Furthermore, volumetric ADC calculated by DWI histogram analysis has shown remarkable reproducibility in patients with other solid neoplasms $(14,15)$. However, the role of noncontrast MRI combined with DWI in the management of GIST has remained unclear since the prolonged overall survival time of GIST patients and the variable appearance of GIST in imaging due to more widely applied adjuvant therapy.

The aim of this study was to investigate whether noncontrast MRI performed with DWI has comparable lesion detection performance to CECT in the detection of GIST, and whether volumetric ADC histogram parameters can identify GIST patients with moderate-to-high risk grades.

\section{Methods}

\section{Patients}

The study protocols were approved by the institutional ethics review board of our institution, and informed consent was waived because of the retrospective nature of this study. A medical record review was conducted from March 2012 to March 2018 using the institutional database, and patients with gastric or small intestinal GISTs were enrolled for qualitative analysis. For quantitative analyses, database research was performed from March 2012 to December 2019. Flow charts depicting the selection and inclusion of subjects for these two parts of the study are provided in Figure 1. The clinical information (age, sex, etc.) for all patients with pathologically confirmed GISTs by surgical or biopsy specimen were recorded by a reader blinded to the results of the imaging analysis.

Consecutive patients with pathologically confirmed GISTs were enrolled for qualitative analysis. CECT and MRI performed both at the time of diagnosis (time interval within 2 weeks) or both during follow-up (time interval within 15 weeks) were included for analysis. GIST patients who underwent curative resection treatment or unresectable GIST patients on targeted therapy were the follow-up cases. All enrolled patients were followed-up for at least 6 months to confirm their disease status. After evaluation by a senior oncologist, including physical and imaging examinations, the status of no evidence of disease was determined, and the patient was grouped as without lesions. The exclusion criteria were the following: (I) changes in treatment strategy between the CECT and MRI examination, (II) patients with an additional non-GIST neoplasm.

For quantitative analysis, the inclusion criteria were as 

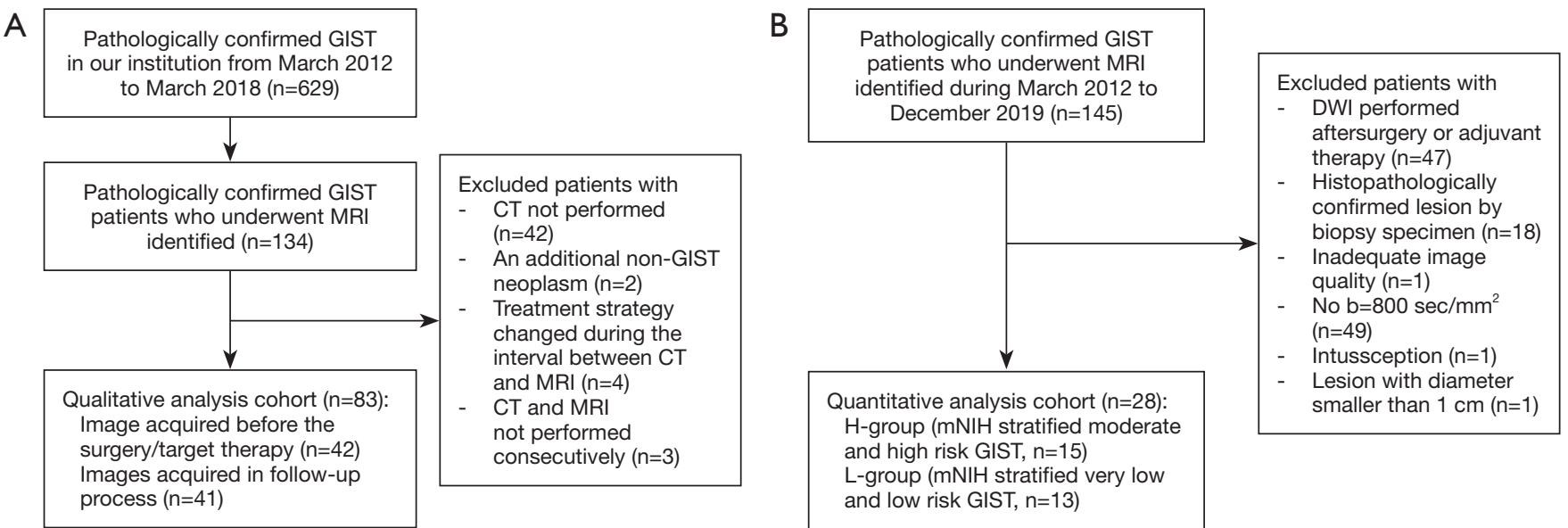

Figure 1 Flowchart of the study population. (A) Flow diagram shows patient inclusion in the qualitative analysis. (B) Flow diagram shows patient selection in the quantitative analysis. GIST, gastrointestinal stromal tumor; MRI, magnetic resonance imaging; CT, computed tomography; mNIH, the modified National Institutes of Health criteria; DWI, diffusion-weighted imaging.

follows: (I) primary GIST patients pathologically confirmed by surgical specimens, (II) DWI performed with a b value of 0 and $800 \mathrm{sec} / \mathrm{mm}^{2}$ on a 3 T MR scanner, (III) no adjuvant therapy performed before DWI. The exclusion criteria were as follows: (I) DWI performed after surgery or adjuvant therapy, (II) insufficient image acquisition and quality, (III) patients with unresectable or metastatic tumors, (VI) patients with intussusception, (V) tumor diameter smaller than $1 \mathrm{~cm}$.

\section{CT and MRI imaging protocols}

Images were acquired with five CT scanners and two $3.0 \mathrm{~T}$ MR scanners (MR system 1 and 2), and all platforms and techniques adhered to the recommendations of the European Society of Gastrointestinal and Abdominal Radiology (ESGAR) and the European Society of Pediatric Radiology (ESPR) (16). The detailed protocols and vendors for CECT and MRI devices are provided in Tables S1,S2. Patients fasted for 4-6 hours, and approximately 1,200-1,500 mL drinking water for CECT and isosmotic mannitol solution for noncontrast MRI were orally administered 30 minutes to 1 hour before scanning. Intravenous contrast agents were only administered for CT. The iodinated contrast agent (Optiray, $350 \mathrm{mgI} / \mathrm{mL}$; Mallinckrodt, Canada) was infused using an automatic power injector (Stellant; Medrad, USA) at a rate of $4 \mathrm{~mL} / \mathrm{s}$, followed by a $20-\mathrm{mL}$ saline solution flush. The total contrast volume varied according to the patient's weight at $1.5 \mathrm{~mL} / \mathrm{kg}$. Using a bolus tracking technique, arterial phase acquisition began 6 seconds after the threshold enhancement of the abdominal aorta reached $120 \mathrm{HU}$. The venous phase started 20-25 seconds after the completion of the arterial phase. The DWI sequence used for quantitative analysis was performed with a b value of 0 and $800 \mathrm{sec} / \mathrm{mm}^{2}$ on the same two $3 \mathrm{~T} \mathrm{MR}$ scanners.

\section{Image interpretation}

Two abdominal radiologists (YQS and XMH, with 10 and 12 years of experience in clinical CT and MRI respectively) independently analyzed CECT and noncontrast MRI in two sessions using the Digital Imaging and Communications in Medicine (DICOM) data of CT and MR image sets of each patient. CT image sets included arterial and venous phase scanning, and multiplanar reconstruction was available to further differentiate lesions from normal tissues. The four pulse sequences listed in Table S2 constitute the MR image sets. Axial and coronal planes for T1-weighted imaging (T1WI), T2WI, fast imaging employing steadystate acquisition (FIESTA), or true fast imaging with steadystate free precession (TRUFI) were available for the MR dataset. The radiologists were blinded to detailed clinical information, and only know that the images were from GIST patients. Each session was performed 4 weeks apart to minimize recall bias. Combined CECT and noncontrast MRI were reviewed by radiologist YQS in a third reading session. 
The radiologists were instructed to record their certainty with regards to the GISTs according to the following scoring system: $1=$ definitely no neoplasm, $2=$ probably no neoplasm, $3=$ indeterminate, $4=$ probably neoplasm, $5=$ definitely neoplasm $(17,18)$. Imaging features for establishing the existence of a single GIST included tumor originating from the muscularis propria layer of the gastrointestinal tract, well-defined margins, intratumoral attenuation or signal pattern related to possible necrosis, cystic degeneration, and hemorrhage. Metastatic lesions were confirmed when more than 1 new mass presented in the liver or peritoneum with or without a lesion located in the gastrointestinal tract. Patients scoring 1 or 2 in the group of patients with no evidence of disease status represented true negatives, and patients scoring 4 or 5 in the group of patients with reference-confirmed existence of GIST lesions represented true positives. Because each individual metastasis could not be pathologically confirmed, a per-lesion analysis was not performed.

DWI was processed with FireVoxel (an open source software, https://wp.nyu.edu/firevoxel/) for quantitative analysis (19). Another two radiologists (ZLZ and JYL, with 3 and 5 years of experience in reading gastrointestinal images, respectively) manually contoured the threedimensional volume of interest along the margin of the entire neoplasm on each slice of the DWI on which the tumor was present $\left(b=800 \mathrm{sec} / \mathrm{mm}^{2}\right)$, excluding the adjacent normal bowel contents and wall. T2-weighted images were used to assist with identifying the contours of the tumors. Examples showing representative volume of interest placement are provided in Figure S1. The ADC map was constructed automatically by using the following monoexponential model:

$$
\mathrm{S}=\mathrm{S}_{0} \times \mathrm{e}^{-\mathrm{b} \times \mathrm{ADC}}
$$

where S and S0 represent the signal intensities with and without diffusion sensitization and $\mathrm{b}$ represents the diffusion-weighting factor. Histogram parameters derived from $\mathrm{DWI}$ were as follows: $\mathrm{ADC}_{\text {mean }}, \mathrm{ADC}_{\text {median }}, \mathrm{ADC}_{\text {min }}$, $\mathrm{ADC}_{\text {max }}$, and $\mathrm{ADC}_{\text {Stdev }} ; 5^{\text {th }}, 10^{\text {th }}, 25^{\text {th }}, 75^{\text {th }}, 90^{\text {th }}$, and $95^{\text {th }}$ percentiles; inhomogeneity, skewness, kurtosis, and entropy. The results of these histogram parameters were calculated as mean values of the two radiologists.

\section{Reference standards}

Diagnostic confirmation was obtained by histopathological examination for cases with CECT and MRI performed before surgery or adjuvant therapy. For cases with CECT and MRI acquired during follow-up, diagnostic confirmation was performed by serial follow-up imaging after the index test in combination with clinical information (20). Clinical follow-up lasted for at least 6 months after performance of the index test for all follow-up cases.

Risk stratification of each primary GIST was defined on the basis of histopathological reports which were graded using the modified National Institutes of Health criteria, including factors such as tumor diameter, site, mitotic index, and presence of rupture at surgery (21). Patients with moderate-to-high risk GISTs were categorized into the high-risk recurrence group (H-group, higher recurrence rate within 5 years) and patients with very-low-and-low risk GISTs were assigned to a low-risk recurrence group (L-group, lower recurrence rate within 5 years) $(22,23)$.

\section{Statistical analysis}

The sensitivity, specificity, and accuracy for identifying GISTs, along with their corresponding $95 \%$ confidence intervals (CIs), were calculated for each imaging technique. Positive and negative predictive values were not computed due to the scoring system defining a score of 3 as neither a true-positive nor a true-negative imaging finding. The differences in sensitivity and specificity between CECT and noncontrast MRI were assessed using the extended paired McNemar test (24). Given a power of 0.95 , a type I error of 0.05 , a total sample size of 83 , and the fixed paired McNemar statistical model, the calculated effect size was moderate, with a value of 0.49 (a range of 0.3 to 0.5 indicated medium effect size). Interobserver agreement between the two readers was assessed using the weighted kappa value. Agreement was defined as follows: $\leq 0.20$, poor; $0.21-0.40$, fair; $0.41-0.60$, moderate; $0.61-0.80$, good; and $>0.80$, excellent.

The volumetric ADC histogram parameters were compared to the modified National Institutes of Health criteria risk grading system. Reliability between the two readers was calculated using the interclass correlation coefficient. For each lesion, mean values of the parameters measured by the two radiologists were analyzed if interobserver agreement was strong (11). After testing the normality of each parameter with the ShapiroWilk test, univariate analysis, including the independent Student's $t$-test or Mann-Whitney $U$ test, was used for the comparison of parameters between the $\mathrm{H}$ - and L-groups. Correlations between histogram parameters and risk grades 
Table 1 Clinicopathological distribution of 83 patients for qualitative analysis

\begin{tabular}{|c|c|c|c|c|c|}
\hline Period of the images acquisition & $\begin{array}{c}\text { Primary } \\
\text { resectable GIST }\end{array}$ & $\begin{array}{c}\text { Unresectable } \\
\text { GIST }\end{array}$ & $\begin{array}{c}\text { Metastatic } \\
\text { lesions }\end{array}$ & $\begin{array}{l}\text { Recurrent } \\
\text { lesions }\end{array}$ & $\begin{array}{c}\text { No evidence } \\
\text { of disease }\end{array}$ \\
\hline Images acquired before surgery or targeted therapy $(n=42)$ & 33 & 3 & $6^{*}$ & 0 & 0 \\
\hline
\end{tabular}

*, 6 cases with metastatic lesions confirmed by biopsy specimens ( 4 cases with metastatic lesions in the liver and 2 cases in the mesenterium);

${ }^{\dagger}, 11$ cases with metastatic lesions ( 6 cases with metastatic lesions in the liver, 4 cases in the mesenterium, 1 case both in the liver and mesenterium). GIST, gastrointestinal stromal tumor.

were evaluated using Spearman's rank correlation. The receiver operator characteristic analyses for univariate variables were performed for histograms that significantly differed between the two different prognostic groups. The optimal cutoff point value of each parameter was calculated by the Youden index. The sensitivity and specificity were estimated for each univariate parameter.

Data management and analyses were performed using SPSS software v.23.0 (IBM Corp., USA) and R software v.3.6.0 (R Project for Statistical Computing). All statistical significance levels were set at a $\mathrm{P}$ value $<0.05$. Effect size was calculated by using $G^{*}$ power software v.3.1.9.4 (25).

\section{Results}

\section{Clinical characteristics}

For the qualitative analysis, there were 55 patients with GIST lesions (28 males, 27 females, with a mean age of $52.82 \pm 10.5)$ and 28 patients with no evidence of disease (16 males, 12 females, with a mean age of $51.29 \pm 10.16$ ). For patients with GIST lesions, we enrolled CECT and noncontrast MRI data from patients with primary gastric GISTs $(n=12)$, small bowel GISTs $(n=21)$, locally advanced GISTs in the small intestine ( $\mathrm{n}=3)$, recurrent GISTs in the stomach $(\mathrm{n}=2)$, and metastatic GISTs $(\mathrm{n}=17)$. The distribution of patients for the qualitative analysis is detailed in Table 1. The distribution of age and sex were comparable between the groups of patients with GISTs and patients with no evidence of disease $(\mathrm{P}=0.523$ and $\mathrm{P}=0.591$, respectively; both $>0.05)$. The CECT and noncontrast MRI in 42 patients (cases with images acquired before the surgery or targeted therapy) were performed with a median of 3 days apart (range from 0 to 11 days). For the 41 patients with CECT and noncontrast MRI performed after surgery, the median time interval between the tests was 16 days (range from 1 to 105 days, 39 cases within 90 days, 2 cases within 105 days).
For the quantitative analysis, 28 patients with a single primary GIST (28 tumors for analysis) were enrolled. There were 11 patients with gastric GISTs and 17 patients with small intestinal GISTs. The mean diameter of the 28 tumors was $4.74 \pm 2.93 \mathrm{~cm}$ (range, $1.5-15 \mathrm{~cm}$ ). The clinicopathological characteristics of these 28 GIST patients are shown in Table 2. According to the modified National Institutes of Health criteria, 12 patients with tumors were categorized as high risk, 3 as intermediate risk, 9 as low risk, and 4 as very low risk. Based on the group criteria above, 15 patients were assigned to the $\mathrm{H}$-group and 13 to the L-group.

\section{Comparison of CECT and noncontrast MRI in the qualitative analysis}

For the 83 patients who underwent matched CECT and noncontrast MRI, detailed scores reflecting the probability of a GIST are shown in Table S3. Subgroup scores of detection performance with CECT and noncontrast MRI based on patients with primary gastric GISTs, primary small bowel GISTs, and patients with metastatic GISTs are presented in Table S4. For both readers, the group of patients with GIST lesions included cases with findings not well-visualized by CECT but more clearly depicted by MRI (Figure 2 and Figure S2).

As previously defined, the true-negatives and truepositives, sensitivity, specificity, and accuracy for detecting GIST lesions with CECT versus noncontrast MRI for reader 1 and reader 2 were calculated and are detailed in Table 3. The extended paired McNemar exact test showed no statistically significant differences overall in the sensitivity and specificity of CECT and noncontrast MRI $(\mathrm{P}=0.607$ by radiologist $1 ; \mathrm{P}=1.000$ by radiologist 2$)$. The concordance of the two readers was good for both CECT (weighted kappa value: 0.691; CI: 0.598-0.783; P<0.001) and noncontrast MRI (weighted kappa value: 0.762 , CI: 0.666-0.858; $\mathrm{P}<0.001)$. 
Table 2 Clinicopathological characteristics of 28 patients with primary GISTs for quantitative analysis

\begin{tabular}{|c|c|c|c|}
\hline Characteristics & $\begin{array}{l}\text { H-group } \\
(n=15)\end{array}$ & $\begin{array}{l}\text { L-group } \\
(n=13)\end{array}$ & $P$ value* \\
\hline Age (year) & $53.07 \pm 10.71$ & $50.54 \pm 12.22$ & 0.565 \\
\hline \multicolumn{4}{|l|}{ Sex } \\
\hline Male & 6 & 8 & \\
\hline Female & 9 & 5 & 0.449 \\
\hline \multicolumn{4}{|l|}{ Tumor site } \\
\hline Stomach & 5 & 6 & \\
\hline Small bowel & 10 & 7 & 0.700 \\
\hline Rupture of the tumor & 0 & 0 & \\
\hline \multicolumn{4}{|l|}{ Tumor diameter $(\mathrm{cm})$} \\
\hline$\leq 5.0$ & 9 & 13 & \\
\hline $5.1-10$ & 5 & 0 & \\
\hline$>10$ & 1 & 0 & $<0.05$ \\
\hline \multicolumn{4}{|l|}{ Mitotic rate (/50 HPF) } \\
\hline$\leq 5$ & 7 & 13 & \\
\hline$>5$ & 8 & 0 & $<0.05$ \\
\hline
\end{tabular}

Age is presented as mean \pm SD. *, data were evaluated by independent $t$-test for continuous variables and chi-square test for categorical variables. H-group: group of patients with higher 5-year recurrence rate (moderate risk and high risk); L-group: group of patients with lower 5-year recurrence rate (very low and low risk). GIST; gastrointestinal stromal tumor. HPF, high power microscope field.

When CECT and noncontrast MRI were analyzed together by radiologist 1 , the number of indeterminate cases decreased from 8 to 3 cases. In the group of patients with GIST lesions, the number of cases with scores of $4-5$ increased from 42 to 50 cases. In the group of patients with no evidence of disease, the number of cases with a score of 1 increased from 4 to 12 cases. Table 3 summarizes the GIST lesion detection performance of CECT and noncontrast MRI.

\section{Correlation between volumetric ADC bistogram parameters and risk grade in the quantitative analysis}

Strong interobserver agreement was reached between the two readers, with interclass correlation coefficients ranging from 0.828 to 0.997 . Table 4 presents the further analysis results of the averaged data of the two readers' measurements for each volumetric ADC parameter between the $\mathrm{H}$ - and $\mathrm{L}$-groups. The $\mathrm{H}$-group showed higher $\mathrm{ADC}_{\text {max }}$ and $\mathrm{ADC}_{\text {stdev }} ; 75^{\text {th }}, 90^{\text {th }}$, and $95^{\text {th }}$ percentiles; and inhomogeneity and entropy values than the L-group. Differences in $\mathrm{ADC}_{\text {min }}, \mathrm{ADC}_{\text {mean }}$, and $\mathrm{ADC}_{\text {median }} ; 5^{\text {th }}, 10^{\text {th }}$, and $25^{\text {th }}$ percentiles; and skewness and kurtosis values between the groups did not reach statistical significance.

\section{Diagnostic performance of volumetric ADC histogram parameters for discrimination of prognosis in the quantitative analysis}

Univariate $\mathrm{ADC}_{\max }$ had the highest area under the curve (0.938) in discriminating between the H- and L-groups of patients with the optimal cutoff value of $2.685 \times 10^{-3} \mathrm{~mm}^{2} / \mathrm{s}$ (Table 5). The corresponding receiver operator characteristic curves for univariate parameters are shown in Figure S3.

\section{Discussion}

This study evaluated the performance of CECT and noncontrast MRI with respect to their sensitivity and specificity for the detection of GIST lesions. The results showed no significant differences in sensitivity and specificity for the detection performance of CECT and noncontrast MRI ( $\mathrm{P}=0.607$ by radiologist $1 ; \mathrm{P}=1.000$ by radiologist 2). In addition, the interobserver variability and the capability of volumetric ADC histogram parameters to discriminate moderate-to-high risk and very-low-to-low risk patients was assessed. Excellent agreement between the two radiologists was reached in the quantitative analysis. Patients with moderate-to-high risk GISTs had overall higher volumetric $\mathrm{ADC}$ parameters $\left(\mathrm{ADC}_{\max }\right.$ value of $\left.3.319 \pm 0.580 \times 10^{-3} \mathrm{~mm}^{2} / \mathrm{s}\right)$ than patients with very-low-tolow risk GISTs $\left(\mathrm{ADC}_{\max }\right.$ value of $2.254 \pm 0.458 \times 10^{-3} \mathrm{~mm}^{2} / \mathrm{s}$ ).

Noncontrast MRI and CECT demonstrated comparable performance for the detection of GIST lesions in our study. The present study extends the use of noncontrast MRI to assess small bowel GIST. GIST in the small intestine shows greater enhancement than other tumors in the small intestine $(26,27)$ and gastric GIST (28). According to these previous studies, GIST should demonstrate avid contrast enhancement and be easily detected on CECT. Nevertheless, noncontrast MRI demonstrated equal detectability with CECT even without contrast media. Noncontrast MRI is advantageous over CECT as there is no concern about radiation exposure in patients. In patients with allergies to iodinated contrast media or renal disease, 



Figure 2 A 52-year-old man with a primary ileal GIST. (A) T2WI showed a round, well-circumscribed small-bowel mass (circle). (B) The mass (circle) demonstrates high signal intensity on DWI. (C) Arterial phase CT images illustrate a poorly defined mildly enhancing ileal lesion (circle). (D) In the venous phase of the CECT, the lesion (circle) remains poorly defined. GIST, gastrointestinal stromal tumor; T2WI, T2-weighted imaging; DWI, diffusion-weighted imaging; CT, computed tomography; CECT, contrast-enhanced computed tomography.

Table 3 Summary of GIST lesion detection performance of CECT and noncontrast MRI

\begin{tabular}{|c|c|c|c|c|c|}
\hline \multirow{2}{*}{ Detection performance } & \multicolumn{2}{|c|}{ Reader 1} & \multicolumn{2}{|c|}{ Reader 2} & \multirow{2}{*}{$\frac{\text { Reader } 1}{\text { Combined CECT and noncontrast MR }}$} \\
\hline & CECT & Noncontrast MRI & CECT & Noncontrast MRI & \\
\hline \multicolumn{6}{|l|}{ Sensitivity (\%) } \\
\hline Value & $76.4(42 / 55)$ & $83.6(46 / 55)$ & $76.4(42 / 55)$ & $81.8(45 / 55)$ & $90.9(50 / 55)$ \\
\hline $95 \% \mathrm{Cl}$ & $(64.77,87.95)$ & $(73.54,93.73)$ & $(64.77,87.95)$ & $(71.3,92.34)$ & $(83.07,98.75)$ \\
\hline Value & $89.3(25 / 28)$ & $89.3(25 / 28)$ & $78.6(22 / 28)$ & $92.9(26 / 28)$ & $89.3(25 / 28)$ \\
\hline $95 \% \mathrm{Cl}$ & $(77.07,100)$ & $(77.07,100)$ & $(62.37,94.77)$ & $(82.69,100)$ & $(77.07,100)$ \\
\hline \multicolumn{6}{|l|}{ Accuracy (\%) } \\
\hline Value & $80.7(67 / 83)$ & $85.5(71 / 83)$ & $77.1(64 / 83)$ & $85.5(71 / 83)$ & $90.4(75 / 83)$ \\
\hline
\end{tabular}

GIST, gastrointestinal stromal tumor; CECT, contrast-enhanced computed tomography; MRI, magnetic resonance imaging; Cl, confidence interval.

noncontrast MRI can be recommended as an alternative safe imaging modality. The lack of intravenous gadolinium contrast also avoids the risk of adverse reactions from contrast injection, the increase in overhead on scanning workflow (having a provider on site in case of contrast reaction), and the introduction of nonessential medications with unknown implications of gadolinium deposition.

The quantitative results of volumetric ADC histogram 
Table 4 Comparisons of volumetric ADC histogram parameters between the H- and L-groups

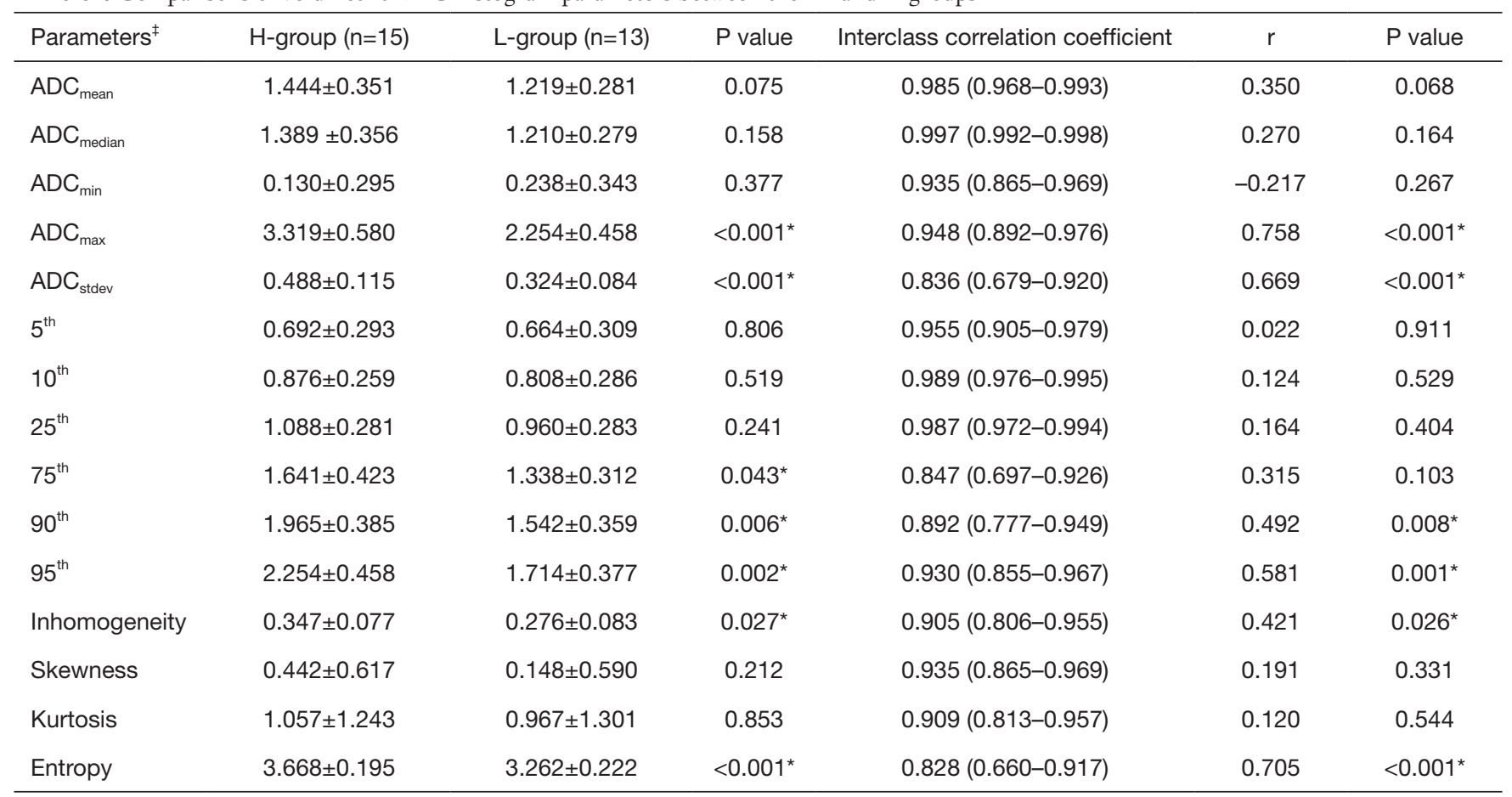

Data are expressed as mean \pm SD, $95 \%$ Cls are shown in parentheses. ${ }^{\ddagger}$, units of $\times 10^{-3} \mathrm{~mm}^{2} / \mathrm{s}$ for all ADC parameters; ${ }^{*}$, values with significant results. H-group: group of patients with higher 5-year recurrence rate (moderate risk and high risk); L-group: group of patients with lower 5-year recurrence rate (very low and low risk). ADC, apparent diffusion coefficient; $A D C_{\text {mean }}$, mean $A D C ; A D C_{\text {median }}$ median $A D C$; $A D C_{\min }$, min $A D C ; A D C_{\max }$, max $A D C ; A D C_{\text {stdev }}$, standard deviation of ADC; $C l$, confidence interval.

Table 5 Area under the curve and cutoff points of ADC histogram parameters for discrimination of the H- and L-groups of patients

\begin{tabular}{lllccc}
\hline Parameters & Area under the curve & Cutoff & Sensitivity (\%) & Specificity (\%) & Youden index \\
\hline $\mathrm{ADC}_{\text {max }}$ & $0.938(0.852-1.000)$ & 2.685 & 84.62 & 93.33 & 0.78 \\
$\mathrm{ADC}_{\text {stdev }}$ & $0.887(0.769-1.000)$ & 0.328 & 61.54 & 61.54 & 73.33 \\
$75^{\text {th }}$ & $0.682(0.482-0.882)$ & 1.475 & 84.62 & 73.33 & 0.61 \\
$90^{\text {th }}$ & $0.785(0.611-0.958)$ & 1.805 & 84.62 & 73.33 & 0.58 \\
$95^{\text {th }}$ & $0.836(0.684-0.988)$ & 2.801 & 53.85 & 100 & 0.58 \\
Inhomogeneity & $0.744(0.550-0.937)$ & 0.256 & 84.62 & 86.67 & 0.54 \\
Entropy & $0.908(0.799-1.000)$ & 3.523 & 0.71 \\
\hline
\end{tabular}

Data in parentheses are $95 \% \mathrm{Cls}$. ADC values are given in units of $\times 10^{-3} \mathrm{~mm}^{2} / \mathrm{s}$. H-group: group of patients with higher 5 -year recurrence rate (moderate risk and high risk); L-group: group of patients with lower 5-year recurrence rate (very low and low risk). ADC, apparent diffusion coefficient; $\mathrm{ADC}_{\text {mean }}$, mean $\mathrm{ADC} ; \mathrm{ADC}_{\text {median }}$, median $\mathrm{ADC} ; \mathrm{Cl}$, confidence interval.

parameters showed moderate sensitivity and specificity for identifying patients with moderate-to-high risk GISTs requiring higher frequency follow-up. Our study demonstrated higher volumetric ADC values with higher risk GISTs as per the modified National Institutes of Health grade. Generally speaking, ADC values reflect the cellularity of the tumor through diffusion of water molecules within tissue, and lower ADC values correspond to the most solid components and high proliferation rates (29). Correspondingly, Zhou et al. and Kang et al. reported that 
lower mean $\mathrm{ADC}$ values are negatively correlated with the modified National Institutes of Health risk stratification of GIST $(11,13)$. However, histogram parameters reflect the distribution of signal intensity over the delineated volume of interest in the tumor, and are independent of absolute measures of signal intensity (30). Underlying molecular diversity is paralleled with the clinicopathological heterogeneity of GISTs. High-risk GISTs have a higher mean size, and pathologically have more heterogeneous composition, such as hemorrhagic foci, central cystic degenerative changes, or necrosis (31). In our study, higher percentile volumetric ADC parameters which corresponded to higher percentiles reflecting cystic or necrotic tissue showed significant differences in discriminating patient groups with different risk grades. Methodological differences may account for the discrepancies with previous studies, as the ADC values in both previous studies were derived from a single slice of tumor, as opposed to the histogram distribution parameters obtained from ADC volumetry in our study. Second, cystic areas or hemorrhage components scattered in the tumor parenchyma represented by the histogram volumetric ADC values such as entropy and inhomogeneity reflected the heterogeneous composition within the tumors. The tumor volume ADC analysis demonstrated strong interobserver reliability in this study, a feature which has been shown in the literature for other neoplasms $(14,32)$. Finally, differences in the study cohort, criteria for risk stratification, b values used, and tumor grade grouping methods may also account for the discrepancies between our results and previous findings.

Previous studies have suggested that dynamic contrast-enhanced MRI could play an important role in characterizing GISTs and assessing therapeutic response (33). The efficacy of DWI for the evaluation of therapeutic response has now been demonstrated by several studies $(34,35)$. Furthermore, DWI texture extracted from patients with metastatic GISTs has the potential to serve as a predictor for overall survival (36). CECT was performed for each patient in our study, and thus, noncontrast MRI with DWI, as opposed to contrast-enhanced MRI, could avoid duplicate infusion of contrast materials, and can be used as a supplemental diagnostic test when needed. Our study presented the complementary roles of noncontrast MRI and CECT for the detection of GIST lesions. Through providing evidence of morphological and functional changes during the entire medical treatment process of GIST, this study suggests that noncontrast MRI with DWI may prove to be an important tool for radiologists and oncologists attempting to formulate an optimal follow-up imaging strategy for GIST.

There are some limitations in this study. First, the relatively small patient population might have caused overestimation of the accuracy of noncontrast MRI in the qualitative analysis, and might have introduced bias in the results of the quantitative analysis. However, the relatively low incidence of GIST and guidelines recommending CECT as the imaging study of choice make it difficult to include patients on a larger scale. Second, overall detection performance rather than separate assessment based on different sites or metastatic lesions was performed in our study, which might have caused bias in the results of the qualitative analysis. Third, the combined reading process of CECT and noncontrast MRI was only performed by 1 radiologist, which meant we were unable to assess the impact of the reader's experience on the results of the performance of combined CECT and noncontrast MRI. Selection bias could not be avoided due to the retrospective nature of this study, leading to overrepresentation of certain populations in this analysis. Per-lesion analysis was not performed in this study.

In conclusion, in the management of patients with GISTs, noncontrast MRI helps detect lesions and predict pathological risk classification. The performance of noncontrast MRI was comparable to that of CECT for the detection of GIST lesions, and noncontrast MRI has a complementary role to CECT in the detection of GISTs. Quantitative noncontrast MRI evaluation through volumetric ADC histogram analysis was useful in predicting modified National Institutes of Health tumor risk grade, offering important prognostic information, and potentially guiding follow-up.

\section{Acknowledgments}

The authors thank Mrs. Fangqin Tan at the technological Department of Radiology for her technical assistance.

Funding: This work was supported by the National Scientific foundation of China (No. 81701657, 81571642, 81771801, and 81801695).

\section{Footnote}

Conflicts of Interest: All authors have completed the ICMJE uniform disclosure form (available at http://dx.doi. org/10.21037/qims-20-578). The authors have no conflicts of interest to declare. 
Ethical Statement: The study was approved by the Institutional Ethics Review Board of Tongji Hospital of Tongji Medical College, Huazhong University of Science and Technology, and informed written consent was waived due to the retrospective nature of this study.

Open Access Statement: This is an Open Access article distributed in accordance with the Creative Commons Attribution-NonCommercial-NoDerivs 4.0 International License (CC BY-NC-ND 4.0), which permits the noncommercial replication and distribution of the article with the strict proviso that no changes or edits are made and the original work is properly cited (including links to both the formal publication through the relevant DOI and the license). See: https://creativecommons.org/licenses/by-nc-nd/4.0/.

\section{References}

1. Reddy P, Boci K, Charbonneau C. The epidemiologic, health-related quality of life, and economic burden of gastrointestinal stromal tumours. J Clin Pharm Ther 2007;32:557-65.

2. Dematteo RP, Ballman KV, Antonescu CR, Maki RG, Pisters PW, Demetri GD, Blackstein ME, Blanke CD, von Mehren M, Brennan MF, Patel S, McCarter MD, Polikoff JA, Tan BR, Owzar K; American College of Surgeons Oncology Group (ACOSOG) Intergroup Adjuvant GIST Study Team. Adjuvant imatinib mesylate after resection of localised, primary gastrointestinal stromal tumour: a randomised, double-blind, placebo-controlled trial. Lancet 2009;373:1097-104.

3. Blanke CD, Demetri GD, von Mehren M, Heinrich MC, Eisenberg B, Fletcher JA, Corless CL, Fletcher CD, Roberts PJ, Heinz D, Wehre E, Nikolova Z, Joensuu H. Long-term results from a randomized phase II trial of standard- versus higher-dose imatinib mesylate for patients with unresectable or metastatic gastrointestinal stromal tumors expressing KIT. J Clin Oncol 2008;26:620-5.

4. Casali PG, Abecassis N, Aro HT, Bauer S, Biagini R, Bielack S, Bonvalot S, Boukovinas I, Bovee JVMG, Brodowicz T, Broto JM, Buonadonna A, De Álava E, Dei Tos AP, Del Muro XG, Dileo P, Eriksson M, Fedenko A, Ferraresi V, Ferrari A, Ferrari S, Frezza AM, Gasperoni S, Gelderblom H, Gil T, Grignani G, Gronchi A, Haas RL, Hassan B, Hohenberger P, Issels R, Joensuu H, Jones RL, Judson I, Jutte P, Kaal S, Kasper B, Kopeckova K, Krákorová DA, Le Cesne A, Lugowska I, Merimsky O, Montemurro M, Pantaleo MA, Piana R, Picci P, Piperno-
Neumann S, Pousa AL, Reichardt P, Robinson MH, Rutkowski P, Safwat AA, Schöffski P, Sleijfer S, Stacchiotti S, Sundby Hall K, Unk M, Van Coevorden F, van der Graaf WTA, Whelan J, Wardelmann E, Zaikova O, Blay JY; ESMO Guidelines Committee and EURACAN. Gastrointestinal stromal tumours: ESMO-EURACAN Clinical Practice Guidelines for diagnosis, treatment and follow-up. Ann Oncol 2018;29:iv68-78.

5. National Comprehensive Cancer Network. NCCN Clinical Practice Guidelines in Oncology, Gastrointestinal Stromal Tumors (GISTs). Version 1.2021. Available online: https://www.nccn.org/professionals/physician_gls/default. aspx (Accessed on 4 November 2020).

6. Maaser C, Sturm A, Vavricka SR, Kucharzik T, Fiorino G, Annese V, Calabrese E, Baumgart DC, Bettenworth D, Borralho Nunes P, Burisch J, Castiglione F, Eliakim R, Ellul P, González-Lama Y, Gordon H, Halligan S, Katsanos K, Kopylov U, Kotze PG, Krustinš E, Laghi A, Limdi JK, Rieder F, Rimola J, Taylor SA, Tolan D, van Rheenen P, Verstockt B, Stoker J; European Crohn's and Colitis Organisation [ECCO] and the European Society of Gastrointestinal and Abdominal Radiology [ESGAR]. ECCO-ESGAR Guideline for Diagnostic Assessment in IBD Part 1: Initial diagnosis, monitoring of known IBD, detection of complications. J Crohns Colitis 2019;13:144-64.

7. Bruining DH, Zimmermann EM, Loftus EV Jr, Sandborn WJ, Sauer CG, Strong SA; Society of Abdominal Radiology Crohn's Disease-Focused Panel. Consensus Recommendations for Evaluation, Interpretation, and Utilization of Computed Tomography and Magnetic Resonance Enterography in Patients With Small Bowel Crohn's Disease. Gastroenterology 2018;154:1172-94.

8. Ryu MH, Lee JL, Chang HM, Kim TW, Kang HJ, Sohn HJ, Lee JS, Kang YK. Patterns of progression in gastrointestinal stromal tumor treated with imatinib mesylate. Jpn J Clin Oncol 2006;36:17-24.

9. Stroszczynski C, Jost D, Reichardt P, Chmelik P, Gaffke G, Kretzschmar A, Schneider U, Felix R, Hohenberger P. Follow-up of gastro-intestinal stromal tumours (GIST) during treatment with imatinib mesylate by abdominal MRI. Eur Radiol 2005;15:2448-56.

10. Yu MH, Lee JM, Baek JH, Han JK, Choi BI. MRI features of gastrointestinal stromal tumors. AJR Am J Roentgenol 2014;203:980-91.

11. Zhou HY, Zhang XM, Zeng NL, Jian SH, Tang W. Use of conventional MR imaging and diffusion-weighted imaging for evaluating the risk grade of gastrointestinal stromal 
tumors. J Magn Reson Imaging 2012;36:1395-401.

12. Yoo J, Kim SH, Han JK. Multiparametric MRI and (18) F-FDG PET features for differentiating gastrointestinal stromal tumors from benign gastric subepithelial lesions. Eur Radiol 2020;30:1634-43.

13. Kang TW, Kim SH, Jang KM, Choi D, Ha SY, Kim KM, Kang WK, Kim MJ. Gastrointestinal stromal tumours: correlation of modified NIH risk stratification with diffusion-weighted MR imaging as an imaging biomarker. Eur J Radiol 2015;84:33-40.

14. Nougaret S, Vargas HA, Lakhman Y, Sudre R, Do RK, Bibeau F, Azria D, Assenat E, Molinari N, Pierredon MA, Rouanet P, Guiu B. Intravoxel Incoherent Motionderived Histogram Metrics for Assessment of Response after Combined Chemotherapy and Radiation Therapy in Rectal Cancer: Initial Experience and Comparison between Single-Section and Volumetric Analyses. Radiology 2016;280:446-54.

15. Kim JY, Kim JJ, Lee JW, Lee NK, Lee G, Kang T, Park H, Son YH, Grimm R. Risk stratification of ductal carcinoma in situ using whole-lesion histogram analysis of the apparent diffusion coefficient. Eur Radiol 2019;29:485-93.

16. Taylor SA, Avni F, Cronin CG, Hoeffel C, Kim SH, Laghi A, Napolitano M, Petit P, Rimola J, Tolan DJ, Torkzad MR, Zappa M, Bhatnagar G, Puylaert CAJ, Stoker J. The first joint ESGAR/ ESPR consensus statement on the technical performance of cross-sectional small bowel and colonic imaging. Eur Radiol 2017;27:2570-82.

17. Masselli G, Polettini E, Casciani E, Bertini L, Vecchioli A, Gualdi G. Small-bowel neoplasms: prospective evaluation of MR enteroclysis. Radiology 2009;251:743-50.

18. Masselli G, Di Tola M, Casciani E, Polettini E, Laghi F, Monti R, Bernieri MG, Gualdi G. Diagnosis of SmallBowel Diseases: Prospective Comparison of MultiDetector Row CT Enterography with MR Enterography. Radiology 2016;279:420-31.

19. Peng Y, Tang H, Meng X, Shen Y, Hu D, Kamel I, Li $Z$. Histological grades of rectal cancer: whole-volume histogram analysis of apparent diffusion coefficient based on reduced field-of-view diffusion-weighted imaging. Quant Imaging Med Surg 2020;10:243-56.

20. Van Weyenberg SJ, Meijerink MR, Jacobs MA, Van der Peet DL, Van Kuijk C, Mulder CJ, Van Waesberghe JH. MR enteroclysis in the diagnosis of small-bowel neoplasms. Radiology 2010;254:765-73.

21. Joensuu H. Risk stratification of patients diagnosed with gastrointestinal stromal tumor. Hum Pathol 2008;39:1411-9.
22. Wada N, Takahashi T, Kurokawa Y, Nakajima K, Masuzawa T, Nakatsuka R, Kawada J, Nishida T, Kimura Y, Tanaka K, Miyazaki Y, Makino T, Yamasaki M, Takiguchi S, Mori M, Doki Y. Appropriate Follow-Up Strategies for Gastrointestinal Stromal Tumor Patients Based on the Analysis of Recurrent Interval and Patterns. Digestion 2017;95:115-21.

23. Joensuu H, Martin-Broto J, Nishida T, Reichardt P, Schoffski P, Maki RG. Follow-up strategies for patients with gastrointestinal stromal tumour treated with or without adjuvant imatinib after surgery. Eur J Cancer 2015;51:1611-7.

24. Hawass NE. Comparing the sensitivities and specificities of two diagnostic procedures performed on the same group of patients. Br J Radiol 1997;70:360-6.

25. Beck TW. The importance of a priori sample size estimation in strength and conditioning research. J Strength Cond Res 2013;27:2323-37.

26. Inoue A, Ota S, Sato S, Nitta N, Shimizu T, Sonoda H, Tani M, Ban H, Inatomi O, Ando A, Kushima R, Murata $\mathrm{K}$. Comparison of characteristic computed tomographic findings of gastrointestinal and non-gastrointestinal stromal tumors in the small intestine. Abdom Radiol (NY) 2019;44:1237-45.

27. Shinya T, Inai R, Tanaka T, Akagi N, Sato S, Yoshino T, Kanazawa S. Small bowel neoplasms: enhancement patterns and differentiation using post-contrast multiphasic multidetector CT. Abdom Radiol (NY) 2017;42:794-801.

28. Inoue A, Ota S, Nitta N, Murata K, Shimizu T, Sonoda H, Tani M, Ban H, Inatomi O, Ando A, Kushima R, Watanabe Y. Difference of computed tomographic characteristic findings between gastric and intestinal gastrointestinal stromal tumors. Jpn J Radiol 2020;38:771-81.

29. Liu S, Zhang Y, Chen L, Guan W, Guan Y, Ge Y, He J, Zhou Z. Whole-lesion apparent diffusion coefficient histogram analysis: significance in $\mathrm{T}$ and $\mathrm{N}$ staging of gastric cancers. BMC Cancer 2017;17:665.

30. Chandarana H, Rosenkrantz AB, Mussi TC, Kim S, Ahmad AA, Raj SD, McMenamy J, Melamed J, Babb JS, Kiefer B, Kiraly AP. Histogram Analysis of Whole-Lesion Enhancement in Differentiating Clear Cell from Papillary Subtype of Renal Cell Cancer. Radiology 2012;265:790-8.

31. Ding H, Yu X, Yu Y, Lao X, Hang C, Gao K, Jia Y, Yan $Z$. Clinical significance of the molecular heterogeneity of gastrointestinal stromal tumors and related research: A systematic review. Oncol Rep 2020;43:751-64.

32. Zou X, Luo Y, Li Z, Hu Y, Li H, Tang H, Shen Y, Hu D, Kamel IR. Volumetric Apparent Diffusion Coefficient 
Histogram Analysis in Differentiating Intrahepatic MassForming Cholangiocarcinoma From Hepatocellular Carcinoma. J Magn Reson Imaging 2019;49:975-83.

33. Consolino L, Longo DL, Sciortino M, Dastru W, Cabodi S, Giovenzana GB, Aime S. Assessing tumor vascularization as a potential biomarker of imatinib resistance in gastrointestinal stromal tumors by dynamic contrast-enhanced magnetic resonance imaging. Gastric Cancer 2017;20:629-39.

34. Tang L, Zhang XP, Sun YS, Shen L, Li J, Qi LP, Cui Y. Gastrointestinal stromal tumors treated with imatinib

Cite this article as: Zhou Z, Lu J, Morelli JN, Hu D, Li Z, Xiao P, Hu X, Shen Y. Utility of noncontrast MRI in the detection and risk grading of gastrointestinal stromal tumor: a comparison with contrast-enhanced CT. Quant Imaging Med Surg 2021;11(6):2453-2464. doi: 10.21037/qims-20-578 mesylate: apparent diffusion coefficient in the evaluation of therapy response in patients. Radiology 2011;258:729-38.

35. Gong NJ, Wong CS, Chu YC, Gu J. Treatment response monitoring in patients with gastrointestinal stromal tumor using diffusion-weighted imaging: preliminary results in comparison with positron emission tomography/computed tomography. NMR Biomed 2013;26:185-92.

36. Fu J, Fang MJ, Dong D, Li J, Sun YS, Tian J, Tang L. Heterogeneity of metastatic gastrointestinal stromal tumor on texture analysis: DWI texture as potential biomarker of overall survival. Eur J Radiol 2020;125:108825. 

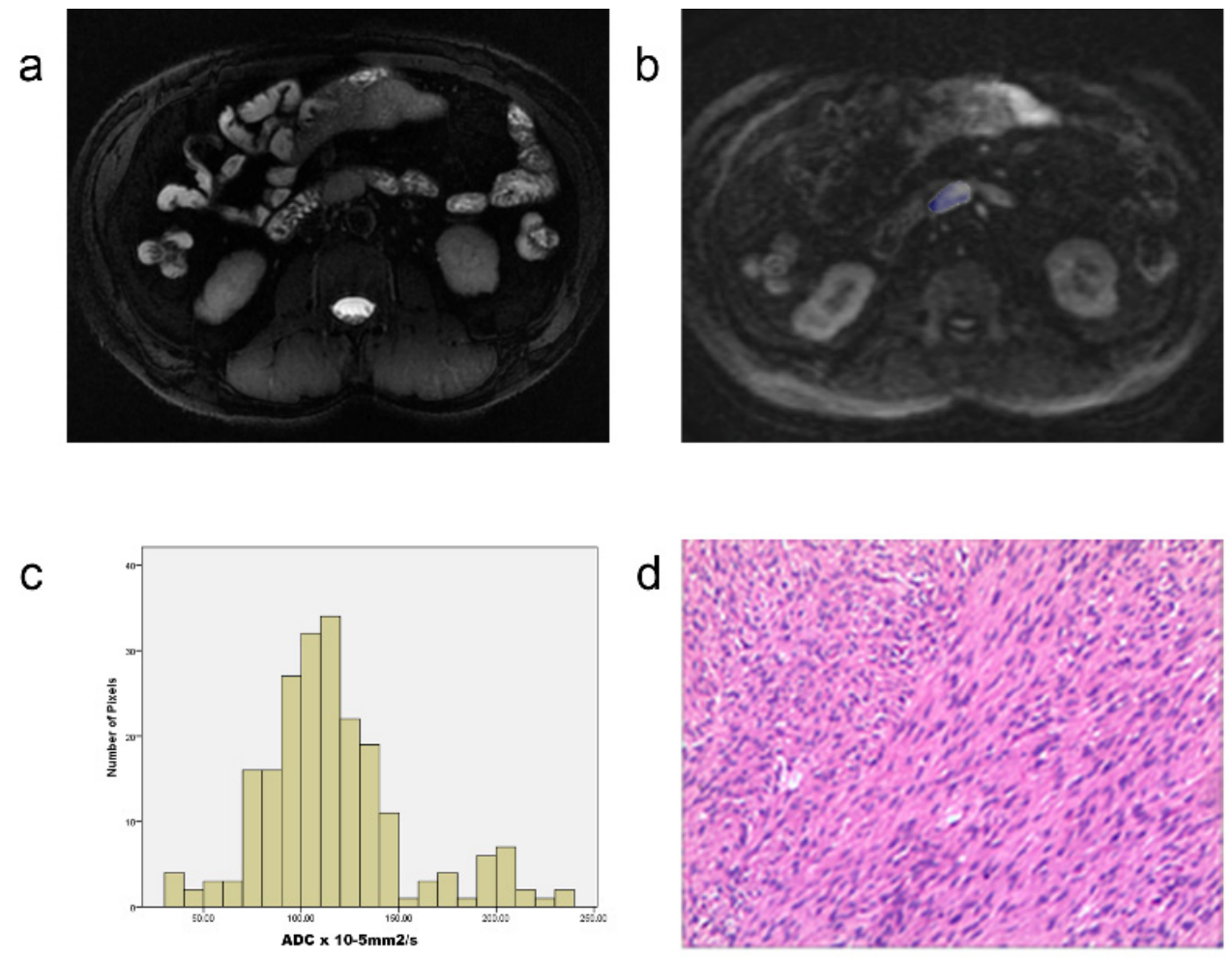

Figure S1 A 53-year-old male with duodenal GIST. (A) T2WI shows a small, oval shaped, well-defined, exophytic mass with isointense signal intensity. (B) Corresponding DWI shows the same lesion with a region of interest placed for ADC value measurement. (C) The histogram of ADC map. (D) Hematoxylin and eosin (H\&E) staining of the tissue demonstrated a low-risk GIST (200×). GIST, gastrointestinal stromal tumor; T2WI, T2-weighted imaging; DWI, diffusion-weighted imaging; ADC, apparent diffusion coefficient.

Table S1 Detailed CT imaging parameters for the five different CT scanners utilized

\begin{tabular}{|c|c|c|c|c|c|}
\hline Parameter & 1 & 2 & 3 & 4 & 5 \\
\hline Tube voltage (kV) & $100-120$ & 100 & 120 & 100 & 120 \\
\hline Matrix & $512 \times 512$ & $512 \times 512$ & $512 \times 512$ & $512 \times 512$ & $512 \times 512$ \\
\hline Detector pitch & $0.984: 1$ & $0.984: 1$ & $1.375: 1$ & 0.993:1 & NA \\
\hline Rotation time (s) & 0.5 & 0.6 & 0.6 & 0.75 & 0.5 \\
\hline Reconstruction thickness (mm) & 1.25 & 1.25 & 1.25 & 1.25 & 1.0 \\
\hline
\end{tabular}

The corresponding serial number representing different CT scanners was displayed as follows: 1= Discovery CT750 HD, GE healthcare, USA; 2= Lightspeed VCT; GE Healthcare, Milwaukee, WI, USA; 3= Lightspeed 16, GE Healthcare, USA; 4= iCT256; Philips, Netherlands; 5= AquilionOne TSX-301A; TOSHIBA, Japan. CT, computed tomography; NA, not available. 
a
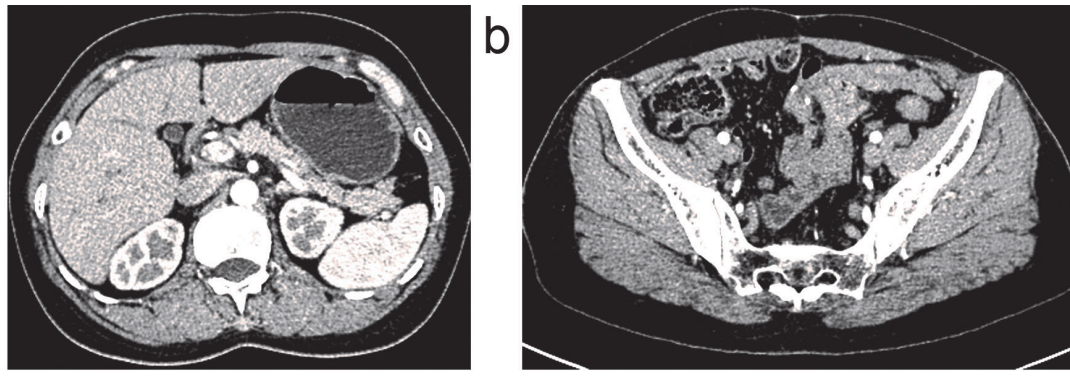

d

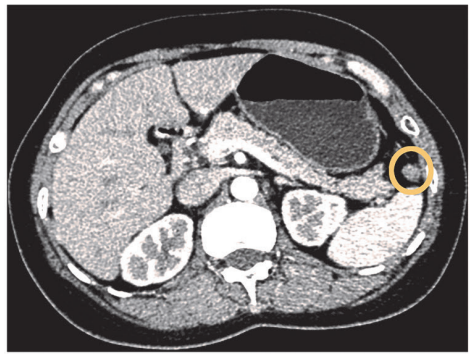

e

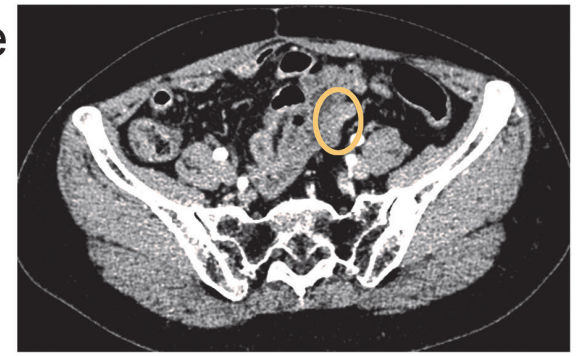

f

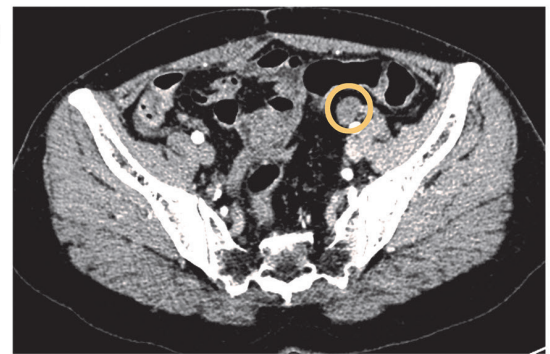

9
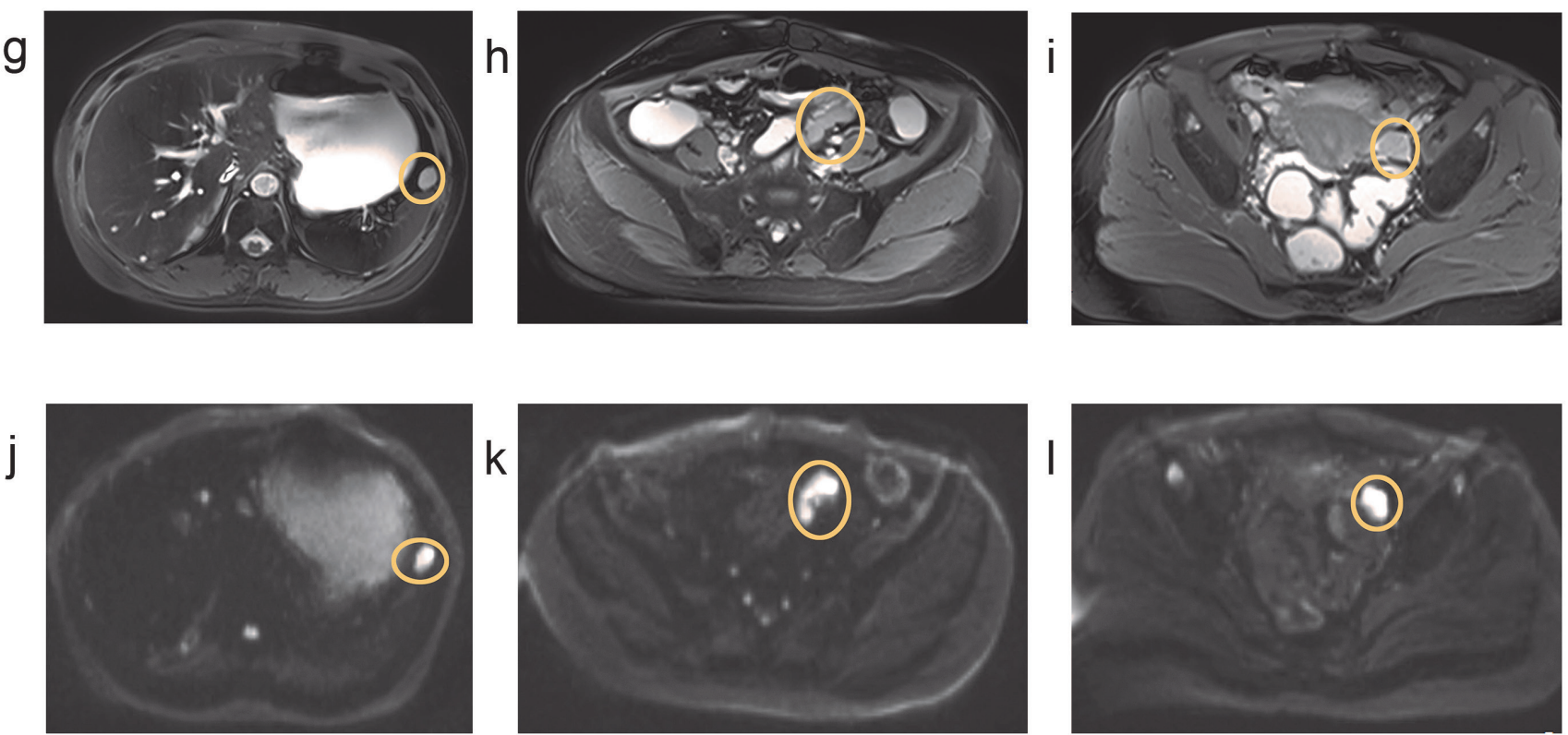

Figure S2 A 55-year-old woman with primary high risk GIST status post resection and 3-year of adjuvant therapy. (A-C) Three slices of a CT during follow-up 3 months after termination of imatinib therapy show no evidence of recurrent tumor. (D-F) The same three slices 6 months after imatinib therapy termination demonstrate three recurrent and metastases lesions (circle) which only mildly enhance, rendering distinction from the surrounding tissues difficult. (G-I) MRI clearly depicts the recurrent tumor (circle). (J-L) All three lesions (circle) have high signal intensity on DWI. GIST, gastrointestinal stromal tumor; CT, computed tomography; MRI, magnetic resonance imaging; DWI, diffusion-weighted imaging. 
Table S2 MRI parameters

\begin{tabular}{|c|c|c|c|c|c|c|c|}
\hline MRI system & Sequences & TR/TE (msec) & NEX & Thickness/gap (mm) & Flip angle & Matrix & Bandwidth \\
\hline \multirow{7}{*}{1} & Coronal & $\mathrm{RC} / 68$ & NA & 4 & $90^{\circ}$ & $288 \times 288$ & 83.33 \\
\hline & Axial & $\mathrm{RC} / 68$ & NA & 5 & $90^{\circ}$ & $288 \times 288$ & 83.33 \\
\hline & T1-weighted 3D LAVA & & & & & & \\
\hline & Axial & 4.0/min Full & 1.00 & 4.6 & $15^{\circ}$ & $260 \times 210$ & 200.00 \\
\hline & FIESTA & & & & & & \\
\hline & Coronal and fs coronal & 3.2/minimum & 1.00 & 4.00 & $45^{\circ}$ & $256 \times 224$ & 166.67 \\
\hline & Axial and fs axial & 3.4/minimum & 1.00 & 5.0 & $45^{\circ}$ & $288 \times 288$ & 125.00 \\
\hline \multirow[t]{8}{*}{2} & T2-weighted HASTE & & & & & & \\
\hline & Coronal and fs coronal & $2,000 / 80$ & 1 & 4.0 & $125^{\circ}$ & $256 \times 240$ & 700 \\
\hline & Axial and fs axial & $2,000 / 81$ & 1 & 5.0 & $112^{\circ}$ & $256 \times 208$ & 575 \\
\hline & T1-weighted 3D VIBE & & & & & & \\
\hline & Coronal & $4.5 / 1.31$ & 1 & 4.0 & $15^{\circ}$ & $288 \times 288$ & 915 \\
\hline & Axial & $4.5 / 1.31$ & 1 & 4.0 & $15^{\circ}$ & $232 \times 288$ & 915 \\
\hline & TRUFI & & & & & & \\
\hline & Coronal & $389.52 / 1.51$ & 1 & 4.0 & $42^{\circ}$ & $512 \times 512$ & 1,500 \\
\hline
\end{tabular}

System 1: HD750 3T (GE Healthcare); system 2: Magnetom Skyra 3.0T (Siemens Healthcare). MRI, magnetic resonance imaging; TR, repetition time; TE, echo time; NEX, number of excitation; SSFSE, single shot fast spin-echo; LAVA, liver acquisition with volume acceleration; FIESTA, fast imaging employing steady state acquisition; DWI, diffusion-weighted image; HASTE, half-fourier acquisition single-shot turbo spin-echo; VIBE, volume interpolated body examination; TRUFI, true fast imaging sequence; 3D, three-dimentional; fs, fat saturated; RC, respiratory cycle; NA, not available. 


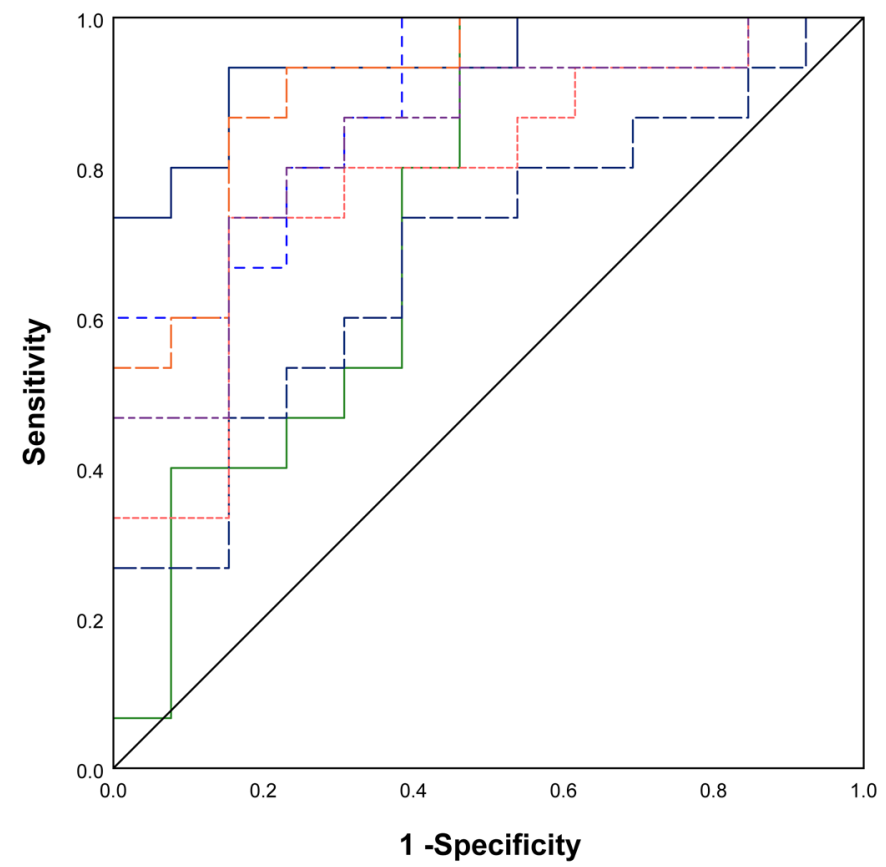

Figure S3 The receiver operator characteristic curve for the univariate ADC histogram parameters of GIST on DWI in distinguishing patients with very low to low risk from moderate to high-risk according to the risk classification defined by the modified National Institutes of Health criteria. ADC, apparent diffusion coefficient; GIST, gastrointestinal stromal tumor; DWI, diffusion-weighted imaging.

Table S3 Diagnostic score of patients with GIST or patients with no evidence of disease for both readers in CECT and non-contrast MRI

\begin{tabular}{|c|c|c|c|c|c|c|c|c|c|c|}
\hline \multirow{3}{*}{ Score } & \multicolumn{5}{|c|}{ Patients with GIST $(n=55)$} & \multicolumn{5}{|c|}{ Patients with no evidence of disease $(n=28)$} \\
\hline & \multicolumn{2}{|c|}{ CECT } & \multicolumn{2}{|c|}{ Non-contrast MRI } & \multirow{2}{*}{$\begin{array}{c}\text { Combined CECT } \\
\text { and non-contrast MRI } \\
(\text { reader } 1)\end{array}$} & \multicolumn{2}{|c|}{ CECT } & \multicolumn{2}{|c|}{ Non-contrast MRI } & \multirow{2}{*}{$\begin{array}{c}\text { Combined CECT } \\
\text { and non-contrast MRI } \\
(\text { reader } 1)\end{array}$} \\
\hline & Reader 1 & Reader 2 & Reader 1 & Reader 2 & & Reader 1 & Reader 2 & Reader 1 & Reader 2 & \\
\hline 1 & 0 & 1 & 0 & 0 & 1 & 4 & 6 & 4 & 7 & 12 \\
\hline 2 & 8 & 6 & 6 & 6 & 2 & 21 & 16 & 21 & 19 & 14 \\
\hline 4 & 12 & 11 & 15 & 9 & 18 & 0 & 2 & 1 & 2 & 0 \\
\hline 5 & 30 & 31 & 31 & 36 & 32 & 0 & 0 & 1 & 0 & 1 \\
\hline
\end{tabular}

Scores 1-5 were given by each of the radiologists for each CT and MRI: $1=$ definitely without neoplasm, 2= probably without neoplasm, $3=$ indeterminate, $4=$ probably with neoplasm, 5= definitely with neoplasm. GIST, gastrointestinal stromal tumor; CECT, contrast enhanced computed tomography; MRI, magnetic resonance imaging. 
Table S4 Detection scores of CECT and non-contrast MRI by reader 1 in primary or metastatic GIST



Scores 1-5 were given by one of the radiologists for each CT and MRI: 1= definitely without neoplasm, 2= probably without neoplasm, 3= indeterminate, 4= probably with neoplasm, 5= definitely with neoplasm. CECT, contrast enhanced computed tomography; MRI, magnetic resonance imaging; GIST, gastrointestinal stromal tumor. 